\title{
Análise do Comportamento Sustentável e Inovador na Intenção Empreendedora
}

\section{Analysis of Sustainable and Innovative Behavior in Entrepreneurial Intention}

\author{
Luis Eduardo Brandão Paiva \\ Universidade Federal do Ceará, UFC, Brasil \\ email:edubrandas@gmail.com
}

\section{Tereza Cristina Batista de Lima}

Universidade Federal do Ceará, UFC, Brasil

email:tcblima@uol.com.br

\section{Sílvia Maria Dias Pedro Rebouças}

Instituto Superior Manuel Teixeira Gomes, ISMAT, Portugal

email:smdpedro@gmail.com

\section{RESUMO}

Este estudo teve como objetivo analisar a influência dos familiares empreendedores e dos constructos comportamentais sustentáveis e inovadores na intenção empreendedora dos estudantes universitários. Tratou-se de uma pesquisa quantitativa, desenvolvida com 285 estudantes de uma Universidade Pública. Foram adotadas as bases teóricas-metodológicas de Liñán e Chen (2009), para a intenção empreendedora e familiares empreendedoras; Gonçalves-Dias et al. (2009), relacionada ao comportamento sustentável; e Foxall e Hackett (1992), referente ao comportamento inovador. Com base na análise fatorial, na regressão logística e nas árvores de classificação e regressão (CART), os resultados constatam: (i) há uma influência dos familiares empreendedores na intenção empreendedora dos estudantes universitários; (ii) há uma influência do comportamento sustentável na intenção empreendedora dos estudantes universitários; e (iii) há uma influência do comportamento inovador na intenção empreendedora dos estudantes universitários. Os resultados buscam contribuir com a literatura empírica e conceitual sobre a inovação e a sustentabilidade no contexto do empreendedorismo.

Palavras-Chave: Comportamento Sustentável. Comportamento Inovador. Intenção Empreendedora.

\section{ABSTRACT}

This study aimed to analyze the influence of the entrepreneurial family and the sustainable and innovative behavioral constructs on the entrepreneurial intention of university students. It was a quantitative research, developed with 285 students of a Public University. The theoretical and methodological bases of Liñán and Chen (2009) were adopted for entrepreneurial intention and entrepreneurial family; Gonçalves-Dias et al. (2009), related to sustainable behavior; and Foxall and Hackett (1992) on innovative behavior. Based on factorial analysis, logistic regression and classification and regression trees (CART), the results show: (i) there is an influence of the entrepreneurial family on the entrepreneurial intention of university students; (ii) there is an influence of sustainable behavior on the entrepreneurial intention of university students; (iii) there is an influence of innovative behavior on the entrepreneurial intent of university students. The results seek to contribute to the empirical and conceptual literature on innovation and sustainability in the context of entrepreneurship.

Key-words: Sustainable Behavior. Innovative Behavior. Entrepreneurial Intention. 


\section{INTRODUÇÃO}

O empreendedorismo é considerado essencial para o desenvolvimento econômico e social de um país (BORGES; FILION; SIMARD, 2012; ACS; SZERB; LLOYD, 2017), podendo ser fundamental para a redução da pobreza e para o desenvolvimento sustentável (DEGEN, 2008). Quanto ao empreendedorismo, destaca-se a intenção empreendedora, considerada essencial e amplamente discutida para compreender e explicar o processo da formação de novos negócios (KRUEGER, 2017), sendo o principal fenômeno para se entender o empreendedorismo como um todo (BIRD, 1988; FAYOLLE; GAILLY, 2015; LIÑÁN; CHEN, 2009; NABI et al., 2018), e pode ser ainda influenciada diretamente pelo contexto familiar (SIEGER; MINOLA, 2017; ZAMPETAKIS et al., 2017), consoante a Teoria do Comportamento Planejado, proposta por Ajzen (1991).

A intenção empreendedora está diretamente relacionada ao empreendedorismo, sendo um processo intrínseco do indivíduo (KAUTONEN; VAN GELDEREN; FINK, 2015; LIÑÁN; CHEN, 2009). Ajzen (1991) acentua que a intenção é anterior ao comportamento real do empreendedorismo, que antecipa a criação ou expansão de um determinado negócio. Carvalho e González (2006), Liñán e Chen (2009), Thompson (2009), Teixeira e Davey (2010), Fayolle e Gailly (2015), Ferreira, Loiola e Gondim (2017) e Paiva et al. (2020) apontam inúmeros modelos baseados na intenção empreendedora, com foco em tentar prever ou antecipar possíveis potenciais empreendedores.

O empreendedor é considerado uma pessoa inovadora, capaz de propor soluções criativas e originais para resolver problemas e tomar decisões em um contexto complexo e de incertezas (SOOMRO; SHAH, 2015). Diante disso, a Teoria da Adaptação-Inovação, proposta por Kirton (1976), é abordada em pesquisas que tentam identificar o comportamento inovador, por meio dos estilos cognitivos (processamento de informações), no contexto do empreendedorismo, a exemplo de Goldsmith e Kerr (1991), Marcati, Guido e Peluso (2008), Ahmed et al. (2010), Wurthmann (2014), Soomro e Shah (2015), Kollmann et al. (2017) e Paiva et al. (2019).
A sustentabilidade também complementa o eixo do empreendedorismo, embasando-se em aspectos relacionados à proteção ambiental, uma vez que os empreendedores podem impactar diretamente ou indiretamente no meio ambiente, tornando-se pertinente que eles incorporem práticas sustentáveis e originais na criação de negócios (BOSZCZOWSKI; TEIXEIRA, 2012). Para que uma empresa seja ambientalmente correta, os empresários devem ter consciência do impacto de suas ações e posturas frente ao meio ambiente (BELZ; BINDER, 2017; DENTCHEV et al., 2016; KUCKERTZ; WAGNER, 2010).

Considerando as abordagens que contextualizaram os temas em questão, levando-se em conta a intenção empreendedora, com base na influência dos familiares na formação dessa intenção, bem como a inovação e a sustentabilidade, ressalta-se o seguinte questionamento para o estudo: como se dá a influência dos familiares, da sustentabilidade e da inovação na intenção empreendedora? Destarte, esta pesquisa tem como objetivo analisar a influência dos familiares empreendedores e dos constructos comportamentais sustentáveis e inovadores na intenção empreendedora dos estudantes universitários.

De modo geral, a contribuição deste estudo justifica-se devido a crescente quantidade de estudos que demonstram o alto índice de intenção empreendedora dos estudantes universitários (FERREIRA; LOIOLA; GONDIM, 2017; LIÑÁN; CHEN, 2009; PAIVA et al., 2020). Assim, torna-se relevante compreender como versa o comportamento inovador e sustentável no contexto do empreendedorismo, buscando-se, desta forma, ampliar a compreensão do alinhamento entre inovação-empreendedorismo-sustentabilidade, levando-se em conta perspectivas intencional e comportamental.

Além desta seção introdutória, o estudo elucida mais quatro seções. A próxima seção trata do desenvolvimento das hipóteses, comtemplando aspectos referentes à intenção empreendedora, baseando-se, também, na influência dos familiares para a formação da intenção empreendedora; e no comportamento sustentável e inovador. Na terceira seção, tem-se os procedimentos metodológicos delineados para a investigação; em seguida, a análise e a discussão dos resultados obtidos. Por fim, são apresentadas as conclusões do estudo, com reflexões para futuras pesquisas. 


\section{DESENVOLVIMENTO DAS HIPÓTESES}

\subsection{Intenção empreendedora}

Estudos e discussões a respeito da intenção empreendedora vêm ganhando visibilidade e relevância a partir, principalmente, das duas últimas décadas do Século XX, como por exemplo os trabalhos de Shapero e Sokol (1982) e Davidsson (1995); e do Século XXI, com Carvalho e González (2006), Liñán e Chen, (2009), Teixeira e Davey (2010), Bae et al. (2014), Fayolle e Gailly (2015), Ferreira, Loiola e Gondim (2017), Krueger (2017), Passaro, Quinto e Thomas (2018) e Paiva et al. (2020).

Além disso, denota-se que as obras seminais de Shapero marcam o ponto crucial de pesquisas referentes à intenção empreendedora (SHAPERO; SOKOL, 1982). Então, pesquisadores do campo de estudos do empreendedorismo reconheceram a importância da intenção empreendedora como peça chave para compreender o processo de criação de uma empresa (BIRD, 1988), bem como os aspectos intrínsecos dos futuros empreendedores (HOCKERTS, 2017; THOMPSON, 2009). Assim, com a evolução da literatura sobre a intenção empreendedora, algumas teorias foram consideradas cruciais para explicar esse fenômeno (AUTIO et al., 2001; GORGIEVSKI et al., 2018).

A Teoria da Ação Racional, de Ajzen e Fishbein (1977), foi a precursora na tentativa de prever e explicar o comportamento e despertou a Teoria da Intenção Empreendedora, de Shapero e Sokol (1982), e a Teoria do Comportamento Planejado (TCP), proposta por Ajzen (1991). Essas teorias caracterizam-se como modelos dominantes que tentam explicar a intenção empreendedora. Desde a década de 1990, a TCP (AJZEN, 1991) é referência para o campo dos estudos relacionados ao empreendedorismo, e com isso é possível, portanto, a partir dessa teoria e de suas abordagens, compreender crenças e comportamentos (DE LEEUW et al., 2015) que são preditores na explicação da intenção empreendedora (GORGIEVSKI et al., 2018; KRUEGER, 2017; LIÑÁN; CHEN, 2009; SOUSA et al., 2017).

Shapero e Sokol (1982) enfatizaram, ainda, a importância da literatura empírica do empreendedo- rismo e das intenções empreendedoras, assim como o reconhecimento de modelos e teorias, como a TCP, que procuram identificar e explicar a intenção empreendedora (KRUEGER; CARSRUD, 1993). Além disso, Vicenzi e Bulgacov (2013), Fayolle e Liñán (2014) e Schlaegel e Koenig (2014) reforçam que a intenção é essencial para se tratar do empreendedorismo. A intenção, antecedente ao comportamento empreendedor, refere-se a uma predisposição para criar ou expandir uma empresa, e pode ainda ser influenciada por inúmeros fatores, a saber: tempo, contexto familiar, inovação, recursos financeiros, competência, entre outros (KHUONG; AN, 2016; TEIXEIRA; DAVEY, 2010).

O contexto familiar é considerado um influenciador na propensão ao empreendedorismo, uma vez que o fato de os pais serem empreendedores pode contribuir à intenção empreendedora do filho (ALTINAY et al., 2012; TEIXEIRA; DAVEY, 2010). Além disso, a partir das investigações realizados por Carvalho e González (2006), Liñán e Chen (2009), Sánchez (2011), Bae et al. (2014), Fayolle e Gailly (2015), Randerson et al. (2015), Wang, Wang e Chen (2017) e Tentama e Paputungan (2019), cabe salientar a forte influência dos pais na intenção empreendedora dos filhos.

Os antecedentes pessoais e familiares podem influenciar na formação da intenção empreendedora do indivíduo (MUELLER; DATO-ON, 2008; WANG; WONG, 2004; ZAMPETAKIS; MOUSTAKIS, 2006). Resultados das investigações empíricas de Teixeira e Davey (2010), Sieger e Minola (2017) e Paiva et al. (2018) constataram que a intenção empreendedora é influenciada diretamente pelo contexto familiar, ou seja, as pessoas que têm empreendedores familiares tendem ao empreendedorismo.

A intenção empreendedora é influenciada pela história familiar, na qual indivíduos que descendem de pais empreendedores tendem a desenvolver intenção e/ou comportamento empreendedor (ZAMPETAKIS; MOUSTAKIS, 2006). Ao levar em conta a perspectiva da Teoria do Comportamento Planejado, Liñán e Chen (2009) e Steinmetz et al. (2016) reforçam que o contexto social em que os indivíduos convivem, a exemplo do familiar, impacta diretamente na formação da intenção empreendedora. Em virtude 
dessas abordagens, é pertinente destacar a seguinte hipótese para este estudo:

H1: há uma influência dos familiares empreendedores na intenção empreendedora dos estudantes universitários.

\subsection{Comportamento sustentável}

O comportamento sustentável, amplamente discutido na Psicologia Ambiental (CORRAL-VERDUGO; PINHEIRO, 1999), refere-se a estudos sobre o meio ambiente (GÜNTHER; PINHEIRO; GUZZO, 2004; SANTOS; PORTO, 2013). As pesquisas relacionadas aos aspectos ambientais são relativamente recentes, e isto possibilita caracterizá-las nos campos multidisciplinar e transdisciplinar, os quais estão, constantemente, desafiando pesquisadores por meio de enfoques múltiplos e diversos (PATO; TAMAYO, 2006), a exemplo do consumo, da preocupação com o lixo, do boicote via consumo, entre outros (GONÇALVES-DIAS et al., 2009; HÖRISCH; KOLLAT; BRIEGER, 2017).

Diante disso, houve um amplo crescimento em pesquisas acadêmicas referentes às questões ambientais, as quais buscam identificar o perfil do indivíduo considerado ecologicamente ou ambientalmente consciente; porém, somente as variáveis demográficas não são suficientes para a investigação deste perfil, tornando necessário, portanto, uma caracterização mais ampla e contextual para a mensuração do comportamento ambiental do indivíduo (ARNOCKY; MILFONT; NICOL, 2014; GÜNTHER; PINHEIRO; GUZZO, 2004; STRAUGHAN; ROBERTS, 1999).

Em relação ao comportamento sustentável, valese mencionar os problemas relacionados ao meio ambiente, uma vez que estão se tornando preocupações de pesquisas referentes aos temas ambientais (GOMES; GORNI; DREHER, 2011; GRISKEVICIUS; CANTÚ; VUGT, 2012), na tentativa de buscar explicações para as causas dos problemas ambientais, além do impacto direto do comportamento do indivíduo no meio ambiente (GONÇALVES-DIAS et al., 2009; SALLIS; OWEN; FISHER, 2015).

O comportamento sustentável, na perspectiva da preocupação do indivíduo com as questões ambientais, alinha-se também às questões sociais e culturais (ARNOCKY; MILFONT; NICOL, 2014; PATO; TAMAYO, 2006; GONÇALVES-DIAS et al., 2009). De acordo com Kuckertz e Wagner (2010), o comportamento empreendedor voltado à sustentabilidade fundamenta-se na avaliação que o empreendedor faz sobre os impactos de suas ações frente ao meio ambiente (GONÇALVES-DIAS et al., 2009).

Ao considerar a perspectiva teórico-metodológica de Straughan e Roberts (1999) e, sobretudo de Gonçalves-Dias et al. (2009), esta pesquisa procura elucidar o comportamento sustentável por meio dos constructos: (i) consumo engajado, consciência dos indivíduos sobre as questões ambientais que envolvem a postura dos fabricantes, bem como na busca de produtos ecologicamente corretos; (ii) preocupação com o lixo, referente ao lixo e limpeza de ambientes domésticos e públicos; (iii) boicote via consumo, relaciona-se ao consumo, contudo na postura dos indivíduos de penalizar produtos ecologicamente incorretos; (iv) mobilização, postura proativa na busca de sensibilizar outros sobre as questões ambientais; e (v) ambiente doméstico, uso cotidiano de recursos naturais, como água e energia elétrica.

Sob a ótica da literatura conceitual e empírica do empreendedorismo e do comportamento sustentável, mostra-se outra hipótese para a pesquisa:

H2: há uma influência do comportamento sustentável na intenção empreendedora dos estudantes universitários.

\subsection{Teoria de Adaptação-Inovação}

A Teoria da Adaptação-Inovação, proposta por Kirton (1976), elucida que as pessoas são capazes de propor soluções criativas e originais para o mercado, pois estão envolvidas diretamente pela criatividade e inovação. Pesquisas empíricas tentam, portanto, retratar a influência da Teoria da Adaptação-Inovação na identificação de possíveis potenciais empreendedores, como as de Ahmed et al. (2010), Kuckertz e Wagner (2010), Wurthmann (2014), Soomro e Shah (2015), Kollmann et al. (2017) e Subotic (2018).

Essa teoria reforça que os estilos cognitivos são as diferenças individuais na preferência de obter, 
organizar e utilizar informações para tomar decisões e promover mudanças organizacionais, pessoais e sociais (KIRTON, 1976; MARCATI; GUIDO; PELUSO, 2008; STUM, 2009). Stum (2009) argumenta, ainda, acerca da influência da globalização nas organizações, na medida em que promovem a capacidade de a pessoa em lidar com as mudanças sociais e mercadológicas. Taylor (1989) elucida que a propensão para o indivíduo tomar decisões e resolver problemas, mediante principalmente as capacidades cognitivas, parte de um comportamento mais inovador do que adaptador.

Kirton (1976) considera dois estilos na Teoria da Adaptação-Inovação: adaptador ("fazer as coisas melhor") e inovador ("fazer as coisas de forma diferente"), sendo um continuum bipolar. Diante dessa perspectiva, vale considerar que essa teoria mostra que os indivíduos mais inovadores são considerados mais criativos, originais e dinâmicos do que os adaptadores, que fazem apenas as coisas melhores, mas não diferentes.

Os líderes inovadores e os empreendedores são capazes de impactar diretamente nos aspectos econômicos, sociais e ambientais de um país. Destarte, é pertinente ressaltar que a Teoria da Adaptação-Inovação procura equilibrar os estilos cognitivos de cada pessoa (Stum, 2009; Taylor, 1989), mediante o uso de escalas para explicar a natureza cognitiva multi-dimensional dos estilos: adaptador e inovador
(FOXALL; HACKETT, 1992; KOLLMANN et al., 2017; TAYLOR, 1989).

Rauch et al. (2009) enfatizam a inovação na literatura mediante a assunção de risco e a criatividade, ou pelo menos à predisposição destes construtos para que, por meio da propensão para inovar, o indivíduo possa agir e tomar decisões diante de incertezas e riscos existentes no mercado. Destaca-se também que, diante de estudos relacionados ao comportamento organizacional associados à propensão para inovar do indivíduo, Kirton (1976), Taylor (1989), Hauser, Tellis e Griffin (2006) e Soomro e Shah (2015) evidenciam o quanto o indivíduo é inovador, criativo, determinado e pode realizar atividades diferentes para a resolução de problemas. Para isso, ele tem que dispor de uma personalidade criativa e inovadora. Destarte, emerge mais uma hipótese para o estudo, ao alinhar o comportamento inovador à intenção empreendedora:

\section{H3: há uma influência do comportamento inovador na intenção empreendedora dos estudantes universitários.}

Diante das hipóteses da pesquisa, que versam sobre a influência dos familiares empreendedores e dos constructos comportamentais sustentáveis e inovadores na intenção empreendedora, pode-se evidenciar o Quadro 1, que indica uma sumarização das hipóteses do estudo, relacionando os constructos e as suas respectivas bases teóricas.

Quadro 1 Síntese das hipóteses

\begin{tabular}{l|l|l}
\multicolumn{1}{c|}{ Constructos } & \multicolumn{1}{c|}{ Hipóteses } & \multicolumn{1}{c}{ Bases teóricas } \\
\hline $\begin{array}{l}\text { Familiares } \\
\text { empreendedores }\end{array}$ & $\begin{array}{l}\mathbf{H}_{1} \text { : há uma influência dos familiares } \\
\text { empreendedores na intenção empreen- } \\
\text { dedora dos estudantes universitários. }\end{array}$ & $\begin{array}{l}\text { Ajzen (1991), Carvalho e González (2006), Liñán e Chen (2009), Teixeira e } \\
\text { Davey (2010), Sánchez (2011), Bae et al. (2014), Fayolle e Gailly (2015), Rand- } \\
\text { erson et al. (2015), Khuong e An (2016), Steinmetz et al. (2016), Wang, Wang } \\
\text { e Chen (2017), Zampetakis et al. (2017) e Gorgievski et al. (2018). }\end{array}$ \\
\hline $\begin{array}{l}\text { Comportamento } \\
\text { sustentável }\end{array}$ & $\begin{array}{l}\mathbf{H}_{2} \text { : há uma influência do comportamen- } \\
\text { to sustentável na intenção empreende- } \\
\text { dora dos estudantes universitários. }\end{array}$ & $\begin{array}{l}\text { Straughan e Roberts (1999), Pato e Tamayo (2006), Gonçalves-Dias et al. } \\
\text { (2009), Kuckertz e Wagner (2010), Arnocky et al. (2014), Sallis, Owen e Fisher } \\
\text { (2015) e Hörisch et al. (2017). }\end{array}$ \\
\hline $\begin{array}{l}\text { Comportamento } \\
\text { inovador }\end{array}$ & $\begin{array}{l}\mathbf{H}_{3} \text { : há uma influência do comportamen- } \\
\text { to inovador na intenção empreendedora } \\
\text { dos estudantes universitários. }\end{array}$ & $\begin{array}{l}\text { Kirton (1976), Foxall e Hackett (1992), Marcati, Guido e Peluso (2008), Stum } \\
\text { (2009), Kuckertz e Wagner (2010), Wurthmann (2014), Soomro e Shah (2015) } \\
\text { e Kollmann et al. (2017). }\end{array}$ \\
\hline
\end{tabular}

Fonte: Elaborado pelos autores. 


\section{PROCEDIMENTOS METODOLÓGICOS}

Esta pesquisa caracteriza-se como quantitativa, de natureza descritiva (COLLIS; HUSSEY, 2005). O método utilizado para obtenção dos dados é um survey (HAIR et al., 2009). A população foi composta por universitários do Curso de Administração de uma Universidade Pública, localizada em um Estado do Nordeste Brasileiro, considerada a melhor Universidade do Norte e Nordeste entre as instituições públicas e privadas, segundo o ranking nacional divulgado em 2017 pelo Ministério da Educação. Optou-se pelo Curso de Administração, pois é uma área de estudos e práticas direcionadas ao empreendedorismo (PAÇO et al., 2011).

O curso de Administração está ligado à gestão e conta com pessoas mais instruídas e propensas ao engajamento frente às questões ambientais e ao empreendedorismo (FERREIRA; LOIOLA; GONDIM, 2017; HAN; KIM, 2010; PAÇO et al., 2011). Na Universidade investigada existem, aproximadamente, 900 estudantes universitários. Diante disso, obteve-se uma amostra de 285 indivíduos, que foi considerada pertinente para representar a população, pois com um nível de confiança de 95\% e margem de erro de $5 \%$, a amostra é satisfatória (corrigida pelo tamanho da população). Os dados foram coletados presencialmente com todos os indivíduos que responderam ao questionário, entre os meses de agosto e dezembro do ano de 2016.

O instrumento da pesquisa foi estruturado mediante um conjunto de itens, constituídos em uma escala Likert de 5 pontos. Para a intenção empreendedora, adotou-se uma análise dicotômica "sim/não" ou se é empreendedor (LIÑÁN; CHEN, 2009); para o comportamento sustentável, variou de 1 "nunca" a 5 "sempre" (GONÇALVES-DIAS et al., 2009); em relação ao comportamento inovador, de 1 "discordo totalmente" a 5 "concordo totalmente" (FOXALL; HACKETT, 1992) (QUADRO 2).

Além desses constructos, mensuram-se os aspectos sociodemográficos dos estudantes universitários, tais como: Gênero (GE); Idade (ID); Estado Civil (ES); e Semestre em que Frequenta (SF). Considera-se, ainda, os Familiares Empreendedores (FE), por meio da experiência dos pais, com o in- tuito de verificar se os estudantes universitários têm pais empreendedores; ou se têm pais que já foram empreendedores, mas não mais; ou se têm pais que nunca foram empreendedores.

Levando-se em conta o tratamento dos dados, utiliza-se a Análise Fatorial Exploratória (AFE) para reduzir o número de variáveis e agrupá-las em constructos, com o tipo de rotação ortogonal Varimax - a mais utilizada quando se busca reduzir o número de variáveis. Além disso, adota-se o critério da variável substituta para cada constructo, sendo a variável com maior carga fatorial, pois é a que tem maior poder de explicação (HAIR et al., 2009).

A Regressão Logística e as Árvores de Classificação e Regressão (CART) permitem analisar a influência dos familiares empreendedores e dos constructos comportamentais sustentáveis e inovadores na intenção empreendedora. Para a análise e o tratamento dos dados, adotou-se o software Statistical Package for the Social Sciences (SPSS) (versão 22.0).

\section{ANÁLISE E DISCUSSÃO DOS RESULTADOS}

\subsection{Perfil da amostra}

A amostra é composta por 285 universitários, em que predominam pessoas com Intenção Empreendedora (IE), correspondentes a 53,3\%; 40\% sem IE; e $6,7 \%$ que já são empreendedores. Embora a maioria da amostra é composta por mulheres $(53,7 \%)$, os homens, de modo geral, demonstram mais IE do que as mulheres ( $51,5 \%$ versus $48,5 \%)$. Verifica-se que os indivíduos com IE, em sua maioria, têm entre 19 e 23 anos, sendo a idade média de 23 anos. Detendo-se base na perspectiva de Liñán e Chen (2009), elucida-se que investigar uma amostra referente à IE dos estudantes universitários é conveniente para pesquisas que pretendem compreender o fenômeno do empreendedorismo como um todo.

Ressalta-se, portanto, dentre os indivíduos que nunca trabalharam, 58,2\% com IE e $41,8 \%$ sem IE. Comparando a IE entre os indivíduos que trabalham nos setores público e privado; quem trabalha em setor privado manifestou ter mais IE (55,5\%) do que quem trabalha em setor público (48,3\%). Para os indivíduos 
Quadro 2 Variáveis do modelo adotado na pesquisa

\begin{tabular}{|c|c|c|}
\hline Constructos & Item & Pergunta \\
\hline $\begin{array}{l}\text { Intenção } \\
\text { Empreendedora }\end{array}$ & IE & Tenho a intenção de empreender nos próximos 5 anos. \\
\hline \multirow{16}{*}{$\begin{array}{l}\text { Comportamento } \\
\text { Sustentável }\end{array}$} & CS1 & Já paguei mais por produtos ambientalmente corretos. \\
\hline & CS2 & Procuro comprar produtos feitos de material reciclado. \\
\hline & CS3 & Já convenci outras pessoas a não comprarem produtos que prejudicam o meio ambiente. \\
\hline & CS4 & As preocupações com o meio ambiente interferem na minha decisão de compra. \\
\hline & CS5 & Leio o rótulo atentamente antes de decidir a compra. \\
\hline & CS6 & Quando não tem lixeira por perto, guardo o papel que não quero mais no bolso. \\
\hline & CS7 & Evito jogar papel no chão. \\
\hline & CS8 & Ajudo a manter as ruas limpas. \\
\hline & $\operatorname{Cs9}$ & Compro produtos de uma empresa mesmo sabendo que ela polui o meio ambiente. \\
\hline & CS10 & Evito usar produto fabricado por empresa que polui o meio ambiente. \\
\hline & CS11 & Falo sobre a importância do meio ambiente com outras pessoas. \\
\hline & CS12 & Mobilizo as pessoas para a conservação dos espaços públicos. \\
\hline & CS13 & Procuro reduzir o meu consumo de recursos naturais escassos. \\
\hline & CS14 & Tomo banho demorado. \\
\hline & CS15 & Fico com a geladeira aberta muito tempo, olhando o que tem dentro. \\
\hline & CS16 & Quando estou em casa, deixo as luzes acesas em ambientes que não são usados. \\
\hline \multirow{13}{*}{$\begin{array}{l}\text { Comportamento } \\
\text { Inovador }\end{array}$} & $\mathrm{Cl} 1$ & Penso em soluções para situações que parecem sem saída. \\
\hline & $\mathrm{Cl} 2$ & Prefiro criar do que melhorar. \\
\hline & $\mathrm{Cl} 3$ & Tenho novas perspectivas para velhos problemas. \\
\hline & $\mathrm{Cl} 4$ & Sou capaz de manter uma posição de desacordo contra o grupo. \\
\hline & $\mathrm{Cl} 5$ & Sou uma pessoa estimulante. \\
\hline & $\mathrm{Cl} 6$ & Tenho ideias originais. \\
\hline & $\mathrm{Cl} 7$ & Compartilho minhas ideias. \\
\hline & $\mathrm{Cl} 8$ & Gosto de variar rotinas já estabelecidas. \\
\hline & $\mathrm{C} 19$ & Prefiro mudança gradual do que radical. \\
\hline & $\mathrm{Cl} 10$ & Lido com várias novas ideias ao mesmo tempo. \\
\hline & $\mathrm{Cl} 11$ & Prefiro trabalhar com um problema de cada vez do que com vários problemas ao mesmo tempo. \\
\hline & $\mathrm{Cl} 12$ & Frequentemente, me arrisco fazendo coisas de forma diferente. \\
\hline & $\mathrm{Cl13}$ & Preciso do estímulo da mudança frequente. \\
\hline
\end{tabular}

Fonte: Elaborado pelos autores.

que nunca trabalharam, destaca-se 58,2\% com IE, e isto difere de Kuckertz e Wagner (2010), pois ressaltaram que os indivíduos com experiências profis- sionais tinham mais IE do que os sem experiências profissionais (TABELA 1).

Em relação à experiência dos pais (familiares empreendedores) e a IE dos estudantes universitários, 
elucida-se que há uma influência dos pais (que são empreendedoras) na IE dos estudantes universitários, em que 152 têm pais empreendedores, e destes, 70 têm IE (68\%) e 23 não tem IE $(22,3)$ e 10 são empreendedores (9,7\%). Por sua vez, dentre os 114 estudantes que não têm pais empreendedores, 45 têm IE $(41,7 \%), 57$ não têm IE $(52,8 \%)$ e 6 são empreendedores (5,6\%) (TABELA 2).
Os resultados de ambas as análises mostraram que há uma associação estatisticamente significante entre experiência profissional e a IE; e entre os familiares empreendedores e a IE, pois o valor $\mathrm{p}$ do qui-quadrado foi 0,000 , que é fortemente aceitável na literatura, visto que existe um patamar mínimo de 0,05 para sua significância na análise. Maroco (2007) enfatiza que o teste do qui-quadrado permite testar se

Tabela 1 Tabulação cruzada entre a experiência profissional e a intenção empreendedora

\begin{tabular}{|c|c|c|c|c|c|}
\hline \multirow{2}{*}{ Experiência profissional } & \multicolumn{3}{|c|}{ Intenção empreendedora } & \multirow{2}{*}{ Total } & \multirow{2}{*}{$\begin{array}{c}\text { Valor } \mathbf{p} \text { do } \\
\text { Qui-quadrado }\end{array}$} \\
\hline & Sim & Não & Empreendedor & & \\
\hline \multirow{2}{*}{ Nunca trabalhou } & 39 & 28 & 0 & 67 & \multirow{10}{*}{0,000} \\
\hline & $58,2 \%$ & $41,8 \%$ & $0,0 \%$ & $100,0 \%$ & \\
\hline \multirow{2}{*}{ Proprietário/sócio de uma empresa } & 8 & 1 & 14 & 23 & \\
\hline & $34,8 \%$ & $4,3 \%$ & $60,9 \%$ & $100,0 \%$ & \\
\hline \multirow{2}{*}{ Trabalha em um setor privado } & 66 & 50 & 3 & 119 & \\
\hline & $55,5 \%$ & $42,0 \%$ & $2,5 \%$ & $100,0 \%$ & \\
\hline \multirow{2}{*}{ Trabalha em um setor público } & 28 & 29 & 1 & 58 & \\
\hline & $48,3 \%$ & $50,0 \%$ & $1,7 \%$ & $100,0 \%$ & \\
\hline \multirow{2}{*}{ Outra situação profissional } & 11 & 6 & 1 & 18 & \\
\hline & $61,1 \%$ & $33,3 \%$ & $5,6 \%$ & $100,0 \%$ & \\
\hline \multirow{2}{*}{ Total } & 152 & 114 & 19 & 285 & \\
\hline & $53,3 \%$ & $40,0 \%$ & $6,7 \%$ & $100,0 \%$ & \\
\hline
\end{tabular}

Fonte: Dados da pesquisa.

Tabela 2 Tabulação cruzada entre os familiares empreendedores e a intenção empreendedora

\begin{tabular}{|c|c|c|c|c|c|}
\hline \multirow{2}{*}{ Experiência dos Pais } & \multicolumn{3}{|c|}{ Intenção Empreendedora } & \multirow[t]{2}{*}{ Total } & \multirow{2}{*}{$\begin{array}{c}\text { Valor } p \text { do } \\
\text { Qui-quadrado }\end{array}$} \\
\hline & Sim & Não & Empreendedor & & \\
\hline \multirow{2}{*}{$\begin{array}{l}\text { Sim, pelo menos um é } \\
\text { empreendedor }\end{array}$} & 70 & 23 & 10 & 103 & \multirow{6}{*}{0,000} \\
\hline & $68,0 \%$ & $22,3 \%$ & $9,7 \%$ & $100,0 \%$ & \\
\hline \multirow{2}{*}{$\begin{array}{l}\text { Não, mas pelo menos um já foi } \\
\text { empreendedor }\end{array}$} & 37 & 34 & 3 & 74 & \\
\hline & $50,0 \%$ & $45,9 \%$ & $4,1 \%$ & $100,0 \%$ & \\
\hline \multirow{2}{*}{$\begin{array}{l}\text { Não, nenhum nunca foi } \\
\text { empreendedor }\end{array}$} & 45 & 57 & 6 & 108 & \\
\hline & $41,7 \%$ & $52,8 \%$ & $5,6 \%$ & $100,0 \%$ & \\
\hline \multirow[t]{2}{*}{ Total } & 152 & 114 & 19 & 285 & \\
\hline & $53,3 \%$ & $40,0 \%$ & $6,7 \%$ & $100,0 \%$ & \\
\hline
\end{tabular}

Fonte: Dados da pesquisa. 
duas ou mais amostras (ou grupos) independentes diferem relativamente a uma determinada característica.

Consoante a essa associação entre os familiares empreendedores e IE, denota-se que existem indícios para se inferir que há uma influência dos familiares empreendedora na IE, e isto aponta para a aceitação da $\mathbf{H}_{1}$ : há uma influência dos familiares empreendedores na intenção empreendedora dos estudantes universitários, o que corrobora aos estudos de Ching e Kitahara (2015), Ferreira, Loiola e Gondim (2017) e Souza, Silveira e Nascimento (2018), realizados também com estudantes universitários brasileiros.

\subsection{Análise fatorial para os constructos comportamentais sustentáveis e inovadores}

Primeiramente, foi realizada a análise fatorial para o comportamental sustentável, levando-se em conta a perspectiva teórico-metodológica de Gonçalves-Dias et al. (2009). Esta análise fatorial apresenta $66,415 \%$ de variância explicada, com o valor do Kaiser-Meyer-Olkin $(\mathrm{KMO}=0,740)$ e o teste de esfericidade de Bartlett (qui-quadrado = 866,843), e isto indica que análise é significante e explicada pelas variáveis em análise (TABELA 3).

Com o critério da variável substituta para cada constructo do comportamento sustentável, verificam-se cinco constructos com as suas respectivas variáveis mais representativas: Consumo engajado - CS4: "As preocupações com o meio ambiente interferem na minha decisão de compra" (carga fatorial de 0,789); Preocupação com o lixo - CS7: "Evito jogar papel no chão" (carga fatorial de 0,903); Ambiente doméstico - CS16: "Quando estou em casa, deixo as luzes acesas em ambientes que não são usados" (carga fatorial de 0,789); Boicote via consumo - CS9: "Compro produtos de uma empresa mesmo sabendo que ela polui o meio ambiente" (carga fatorial de 0,772) e Mobilização - CS12: "Mobilizo as pessoas para a conservação dos espaços públicos" (carga fatorial de 0,824).

Para a outra análise, considerando o comportamento inovador, aponta-se $67,429 \%$ de variância explicada para a análise, com valor do Kaiser-Meyer-Olkin (KMO $=0,601)$ e teste de esfericidade de Bartlett (qui-quadrado $=196,423$ ) indicando que a análise também é significante (TABELA 4).

Tabela 3 Análise fatorial para o comportamento sustentável

\begin{tabular}{|c|c|c|c|c|c|c|}
\hline \multirow[b]{2}{*}{ Variáveis } & \multicolumn{5}{|c|}{ Cargas fatoriais } & \multirow[b]{2}{*}{ Comunalidades } \\
\hline & $\begin{array}{c}\text { Consumo enga- } \\
\text { jado }\end{array}$ & $\begin{array}{c}\text { Preocupação } \\
\text { com o lixo }\end{array}$ & $\begin{array}{l}\text { Ambiente do- } \\
\text { méstico }\end{array}$ & $\begin{array}{l}\text { Boicote via } \\
\text { consumo }\end{array}$ & Mobilização & \\
\hline $\mathrm{CS} 1$ &, 771 & & & & &, 641 \\
\hline $\mathrm{CS} 2$ &, 736 & & & & & 648 \\
\hline CS4 & 789 & & & & & 701 \\
\hline CS5 & ,703 & & & & &, 567 \\
\hline CS6 & &, 850 & & & &, 741 \\
\hline CS7 & & 903 & & & & ,829 \\
\hline CS8 & & ,780 & & & &, 638 \\
\hline CS15 & & & ,780 & & & 671 \\
\hline CS16 & & & ,789 & & & 661 \\
\hline CS9 & & & & 772 & & 624 \\
\hline CS14 & & & & ,739 & &, 628 \\
\hline CS12 & & & & & ,824 & 717 \\
\hline $\mathrm{CS} 13$ & & & & &, 640 &, 567 \\
\hline
\end{tabular}

Fonte: Dados da pesquisa. 
Tabela 4 Análise fatorial para o comportamento inovador

\begin{tabular}{|c|c|c|c|c|c|}
\hline \multirow[b]{2}{*}{ Variáveis } & \multicolumn{4}{|c|}{ Cargas fatoriais } & \multirow[b]{2}{*}{ Comunalidades } \\
\hline & $\begin{array}{l}\text { Adequação à } \\
\text { originalidade }\end{array}$ & $\begin{array}{c}\text { Eficiência nos } \\
\text { detalhes }\end{array}$ & $\begin{array}{c}\text { Preferência ao } \\
\text { dinamismo e à } \\
\text { criatividade }\end{array}$ & $\begin{array}{c}\text { Preferência pela } \\
\text { mudança }\end{array}$ & \\
\hline $\mathrm{Cl} 1$ & ,797 & & & &, 658 \\
\hline $\mathrm{Cl} 3$ & 802 & & & & 655 \\
\hline $\mathrm{Cl9}$ & & 820 & & & ,707 \\
\hline $\mathrm{Cl} 11$ & &, 807 & & & ,695 \\
\hline $\mathrm{Cl} 5$ & & & ,707 & &, 631 \\
\hline $\mathrm{Cl} 7$ & & & ,856 & & 765 \\
\hline $\mathrm{Cl} 8$ & & & &, 618 &, 550 \\
\hline $\mathrm{Cl} 13$ & & & & ,841 & ,732 \\
\hline
\end{tabular}

Fonte: Dados da pesquisa.

Diante disso, adotam-se as variáveis com maiores cargas fatoriais em cada constructo, levando-se em conta Foxall e Hackett (1992): Adequação à originalidade - CI3: "Tenho novas perspectivas para velhos problemas" (carga fatorial de 0,802 ); Eficiência nos detalhes - CI9: "Prefiro mudança gradual do que radical" (carga fatorial de 0,820); Preferência ao dinamismo e à criatividade - CI7: "Compartilho minhas ideias” (carga fatorial de 0,856); e Preferência pela mudança - CI13: "Preciso do estímulo da mudança frequente" (carga fatorial de 0,841).

\subsection{Análise da regressão logística e do CART}

Diante das estruturas fatoriais da análise fatorial exploratória, são considerados nove constructos comportamentais, por meio das suas variáveis substitutas que constituem cada constructo. Adotou-se a regressão logística para analisar a influência dos familiares empreendedores, dos constructos comportamentais sustentáveis e inovadores, bem como das variáveis relacionadas ao perfil da amostra (variáveis independentes) na IE (variável dependente) (TABELA 5).

O modelo de regressão tem eficiência de 81,9\% para explicar a IE, expressando $\mathrm{R}^{2}$ de Nagelkerke de 0,324 , com o valor da razão de verossimilhança ( $p$ de 0,000$)$, confirmando-se a viabilidade do modelo aplicado.
Em relação ao comportamento sustentável, retrata-se a influência da mobilização na IE (valor p de $0,029, \mathrm{~B}$ de 0,260 ), que permite inferir que as pessoas que se preocupam mais em mobilizar outras pessoas quanto à importância da conservação dos espaços públicos e do meio ambiente apresentam, por sua vez, mais propensão à IE, quando comparadas com as pessoas sem essa preocupação, fato indicativo para confirmar $\mathbf{H}_{2}$ : há uma influência do comportamento sustentável na intenção empreendedora dos estudantes universitários, coincidindo com as pesquisas de Tilley e Young (2009), Kuckertz e Wagner (2010) e Boszczowski e Teixeira (2012).

No tocante ao comportamento inovador, verificam-se influências das variáveis adequação à originalidade (valor p de $0,001, \mathrm{~B}$ de 0,657 ) e preferência pela mudança (valor p de $0,007, B$ de 0,434 ) na IE. Assim, os universitários mais originais, com novas perspectivas sobre os problemas, têm $92,8 \%$ de chance de terem IE $(\operatorname{Exp}(B)=1,928)$; e os que preferem mudanças constantes têm $54,3 \%$ de chance de terem IE $(\operatorname{Exp}(B)=1,543)$, e isto possibilita aceitar $\mathbf{H}_{3}$ : há uma influência do comportamento inovador na intenção empreendedora dos estudantes universitários, o que corrobora a Marcati, Guido e Peluso (2008), Ahmed et al. (2010), Wurthmann (2014), Soomro e Shah (2015) e Kollmann et al. (2017).

Evidencia-se a influência do familiares empreendedores na IE dos universitários, com a variável experiência dos pais (pelo menos um é empreende- 
Tabela 5 Análise da regressão logística das variáveis do modelo

\begin{tabular}{|c|c|c|c|}
\hline Variáveis & B & Sig. & $\operatorname{Exp}(B)$ \\
\hline Consumo engajado &,- 099 &, 556 & 906 \\
\hline Preocupação com o lixo &,- 598 &, 102 &, 550 \\
\hline Ambiente doméstico &,- 227 &, 140 & ,797 \\
\hline Boicote via consumo & ,096 &, 581 & 1,101 \\
\hline Mobilização & ,260 & ,029 & 1,296 \\
\hline Adequação à originalidade & ,657 & ,001 & 1,928 \\
\hline Eficiência nos detalhes &,- 083 &, 563 & 920 \\
\hline Preferência ao dinamismo e à criatividade &,- 097 &, 579 & 908 \\
\hline Preferência pela mudança & ,434 & ,007 & 1,543 \\
\hline Sexo (masculino) & 688 & 025 & 1,989 \\
\hline Idade &,- 039 & 288 & 962 \\
\hline Estado Civil &,- 217 &, 646 &, 805 \\
\hline Semestre &,- 080 &, 177 & 923 \\
\hline Experiência profissional (Nunca Trabalhou) &,- 620 & ,341 &, 538 \\
\hline Experiência profissional (Trabalha no setor privado) &,- 369 &, 551 & ,692 \\
\hline Experiência profissional (Trabalha no setor público) &,- 513 &, 447 &, 599 \\
\hline Experiência dos Pais (Pelo menos um é empreendedor) & 1,483 & ,000 & 4,406 \\
\hline Experiência dos Pais (Pelo menos um já foi empreendedor) &, 482 &, 178 & 1,619 \\
\hline Constante & $-2,142$ & 969 &, 117 \\
\hline
\end{tabular}

Fonte: Dados da pesquisa.

dor), valor p de 0,000 , B de 1,483 e $\operatorname{Exp}(B)=4,406$, ressaltando-se que os indivíduos com pais empreendedores têm quatro vezes a mais de chances de terem IE do que os que não têm pais empreendedores, e isto permite aceitar $\mathbf{H}_{1}$ : há uma influência dos familiares empreendedores na intenção empreendedora dos estudantes universitários, consoante a Randerson et al. (2015), Wang, Wang e Chen (2017), Sieger e Minola, 2017 e Zampetakis et al. (2017). Além disso, vale destacar que os homens têm $98,8 \%$ a mais de chances de terem IE, quando comparados com as mulheres $(\operatorname{Exp}(B)=1,989)$, valor $\mathrm{p}$ de 0,025 e B de 0,688 , mostrando, então, que as mulheres estão menos propensas ao empreendedorismo.

Outro método adotado foi o CART, que se fundamenta na execução de partições binárias sucessivas de uma amostra, detendo-se, portanto, base nos resultados amostrados das covariáveis, e isto procura constituições de subamostras internas homogêneas (TACONELI; ZOCCHI; DIAS, 2008). Os nós são subconjuntos da aplicação das regras de divisão dos dados em relação a variável dependente. A raiz da árvore de classificação se caracteriza por ser o primeiro nó, correspondendo ao conjunto de dados; e as folhas são consideradas os nós terminais (FERREIRA; SOARES; CRUZ, 2001), conforme observa-se pela Figura 1.

Diante do primeiro grupo formado pelo CART, verifica-se influência dos constructos comportamentais sustentáveis - mobilização e ambiente doméstico; inovadores - eficiência nos detalhes; e dos familiares empreendedores (importância normalizada 100\%) na IE dos estudantes universitários. Esse grupo indica que universitários com IE revelaram-se, por sua vez, eficientes nos detalhes; com hábitos de mobilizar outras pessoas no tocante à importância ambiental; com 
Figura 1 Árvores de classificação e regressão - CART

IE

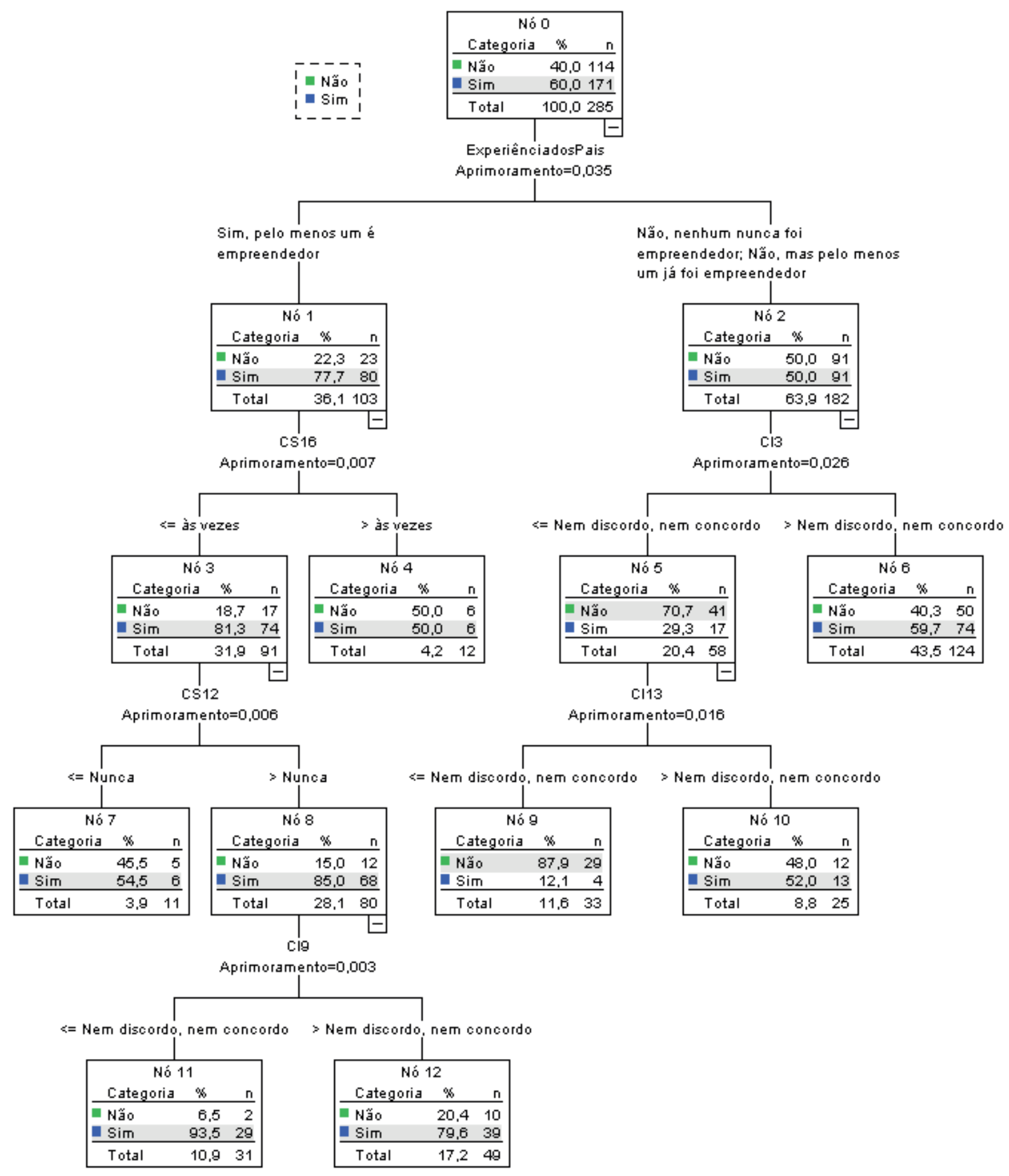

Fonte: Dados da pesquisa. 
costumes de se preocupar com o ambiente doméstico, que buscam não deixar as luzes acessa quando não estão em casa; e ainda são filhos de pais empreendedores.

Outro grupo formado, considerando também os universitários com IE, pode ser retratado pelas variáveis: adequação à originalidade; e pais que já foram empreendedores, porém não mais. Portanto, cabe enfatizar que os estudantes universitários com IE estão concentrados no estilo cognitivo inovador, capazes de propor soluções criativas, originais e inovadoras, coincidindo com a Teoria da Adaptação-Inovação, proposta por Kirton (1979), e isto corrobora aos achados de Marcati, Guido e Peluso (2008), Wurthmann (2014) e Soomro e Shah (2015). No entanto, o grupo dos estudantes universitários que não preferem mudanças; não se consideram originais; e que não têm pais empreendedores; não demonstrou ter IE.

Ao considerar essas análises, é pertinente elucidar a influência do contexto familiar na formação da IE dos indivíduos, que se alinha à Teoria do Comportamento Planejado, de Ajzen (1991), referente à influência do contexto social (familiares) na formação da IE, e isto corrobora aos resultados empíricos das pesquisas de Carvalho e González (2006), Teixeira e Davey (2010), Steinmetz et al. (2016) e Sieger e Minola (2017). De modo geral, foram encontradas influências dos constructos comportamentais sustentáveis (KUCKERTZ; WAGNER, 2010); inovadores (MARCATI; GUIDO; PELUSO, 2008); e dos familiares empreendedores (WANG; WANG; CHEN, 2017) na intenção empreendedora (LIÑÁN; CHEN, 2009).

De acordo com os resultados da regressão logística e do CART, por meio das análises adotadas e discutidas, constataram-se evidências suficientes para a aceitação das hipóteses do estudo: $\mathbf{H}_{1}, \mathbf{H}_{2}$ e $\mathbf{H}_{3}$, conforme observa-se pelo Quadro 3 uma síntese dos valores observados e esperados das hipóteses.
Esta pesquisa torna-se pertinente por vislumbrar um panorama acerca da influência dos familiares empreendedores e dos constructos comportamentais sustentáveis e inovadores na IE dos universitários brasileiros - o que confere uma ampliação para a literatura empírica referente ao alinhamento entre o empreendedorismo, a inovação e a sustentabilidade.

\section{CONCLUSÕES}

Este estudo teve como objetivo analisar a influência dos familiares empreendedores e dos constructos comportamentais sustentáveis e inovadores na intenção empreendedora dos estudantes universitários. A metodologia aplicada se mostrou pertinente para atender ao objetivo proposto da pesquisa, concentrando-se, sobretudo no modelo da regressão logística e no método CART.

Os resultados permitiram apontar que existe influência do contexto familiar na intenção empreendedora dos universitários - ou seja, os que têm pais empreendedores estão mais propensos a terem IE do que os que não têm pais empreendedores, e isto coincidiu com a ótica da Teoria do Comportamento Planejado, de Ajzen (1991), utilizada como modelo teórico dominante para compreender e explicar o empreendedorismo, conforme enfatizaram, em suas pesquisas empíricas, Liñán e Chen (2009), Schlaegel e Koenig (2014), Kautonen, Van Gelderen e Fink (2015) e Zampetakis et al. (2017).

Verificou-se, ainda, que o comportamento sustentável teve influência na IE dos universitários, principalmente pelo constructo mobilização, indicando que quanto mais o indivíduo mobiliza outros no que diz respeito à importância e conservação ambiental, maior a possibilidade de ter IE; e os que tiveram preocupações evidentes quanto ao ambiente

Quadro 3 Síntese das hipóteses

\begin{tabular}{c|c|c|c}
\hline Hipótese & Constructo & Valor esperado da hipótese & Valor observado da hipótese \\
\hline $\mathbf{H}_{1}$ & Familiares Empreendedores & Influência positiva na IE & Influência positiva \\
\hline $\mathbf{H}_{2}$ & Comportamento Sustentável & Influência positiva na IE & Influência positiva \\
\hline $\mathbf{H}_{3}$ & Comportamento Inovador & Influência positiva na IE & Influência positiva \\
\hline
\end{tabular}

Fonte: Elaborado pelos autores. 
doméstico, como o consumo de energia, também tiveram maiores probabilidades de terem IE. Essa análise elucidou a relevância das preocupações ambientais no empreendedorismo, relembrando que o empreendedor, como impacta na economia, na sociedade e no meio ambiente, deve estar ciente da importância de suas ações e posturas frente às questões ambientais.

Ao considerar a Teoria da Adptação-Inovação, de Kirton (1976), utilizada para prever potenciais empreendedores, pôde-se constatar que os estudantes universitários com IE apresentaram, por sua vez, comportamentos mais voltados para a inovação, considerando-se, então, a perspectiva de que um empreendedor é alguém inovador, capaz de propor soluções criativas e originais para o mercado.

Wurthmann (2014) e Soomro e Shah (2015), ao realizarem pesquisas empíricas sobre a identificação de potenciais empreendedores, bem como da inovação na intenção em iniciar novos negócios, constataram que a Teoria do Comportamento Planejado, de Ajzen (1991), bem como a Teoria da Adaptação-Inovação, de Kirton (1976), são pertinentes para identificar potenciais empreendedores, e isto foi evidenciado neste estudo - que permitiu analisar, além da sustentabilidade, a influência do contexto familiar e da inovação na IE.

Ao levar em conta as dificuldades atuais, como crises econômicas, sociais e ambientais, o empreendedorismo é uma alternativa para que a pessoa se insira no mundo do trabalho e contribua para a sociedade. Como a IE é relevante na atualidade, este estudo teve como principal diretriz contribuir para fomentar o incentivo de políticas e práticas direcionadas às Instituições de Ensino Superior (IES), com a expectativa de que as IES possam incorporar estudos e práticas ambientais, inovadoras e sociais alinhadas ao empreendedorismo - a fim de potencializar benefícios sociais e ambientais gerados pelos empreendedores - os quais proporcionam emprego e renda para a sociedade.

Embora a literatura sobre intenção empreendedora seja ampla, tanto nos bancos de dados nacionais como internacionais, não foram encontrados estudos que possibilitassem o alinhamento da inovação e da sustentabilidade na intenção empreendedora dos estudantes universitários, o que é fundamental para sobrelevar um caráter inovador para esta pesquisa.
Esta pesquisa detém algumas limitações que não foram suplantadas, como a investigação em uma única universidade, em um país e não considerado aspectos longitudinais. Portanto, recomenda-se, para futuras pesquisas, alinhar a sustentabilidade e a inovação na IE entre diferentes cursos, com outras universidades e, além disso, realizar um comparativo entre países, a fim de ampliar, ainda mais, a sustentabilidade e a inovação no contexto do empreendedorismo.

\section{REFERÊNCIAS}

ACS, Z. J.; SZERB, L.; LLOYD, A. The global entrepreneurship and development index. In: Global Entrepreneurship and Development Index 2017. Springer, Cham, 2017. p. 29-53.

AHMED, I.; NAWAZ, M. M.; AHMAD, Z.; SHAUKAT, M. Z.; USMAN, A.; REHMAN, W. U.; AHMED, N. Determinants of students' entrepreneurial career intentions: Evidence from business graduates. European Journal of Social Sciences, v. 15, n. 2, p. 14-22, 2010.

AJZEN, I. The theory of planned behavior. Organizational behavior and human decision processes, v. 50, n. 2, p. 179-211, 1991.

AJZEN, I.; FISHBEIN, M. Attitude-behavior relations: A theoretical analysis and review of empirical research. Psychological Bulletin, v. 84, n. 5, p. 888, 1977.

ALTINAY, L.; MADANOGLU, M.; DANIELE, R.; LASHLEY, C. The influence of family tradition and psychological traits on entrepreneurial intention. International Journal of hospitality management, v. 31, n. 2, p. 489-499, 2012.

ARNOCKY, S.; MILFONT, T.L.; NICOL, J. R. Time perspective and sustainable behavior: Evidence for the distinction between consideration of immediate and future consequences. Environment and Behavior, v. 46, n. 5, p. 556-582, 2014 
AUTIO, E.; KEELEY, R.; KLOFSTEN, M., GC PARKER, G.; HAY, M. Entrepreneurial intent among students in Scandinavia and in the USA. Enterprise and Innovation Management Studies, v. 2, n. 2, p. 145-160, 2001.

BELZ, F. M.; BINDER, J. K. Sustainable entrepreneurship: A convergent process model. Business Strategy and the Environment, v. 26, n. 1, p. 1-17, 2017.

BAE, T. J.; QIAN, S.; MIAO, C.; FIET, J. O. The Relationship between Entrepreneurship Education and Entrepreneurial Intentions: A Meta-Analytic Review. Entrepreneurship Theory and Practice, v. 38, n. 2, p. 217-254, 2014.

BIRD, B. Implementing entrepreneurial ideas: The case for intention. Academy of Management Review, v. 13, n. 3, p. 442-453, 1988.

BORGES, C.; FILION, L J.; SIMARD, G. Criação de empresas: um processo mais rápido e fácil resulta em empresas de melhor desempenho? Revista de Ciências da Administração, v. 15, n. 35, p. 196-207, 2013.

BOSZCZOWSKI, A. K.; TEIXEIRA, R. M. O empreendedorismo sustentável e o processo empreendedor: em busca de oportunidades de novos negócios como solução para problemas sociais e ambientais. Revista Economia \& Gestão, v. 12, n. 29, p. 141-168, 2012.

CARVAlHO, P. M. R.; GONZÁLEZ, L. Modelo explicativo sobre a intenção empreendedora. Comportamento Organizacional e Gestão, v. 12, n. 1, p. 43-65, 2006.

CHING, H. Y; KITAHARA, J. R. Propensão a empreender: uma investigação quantitativa baseada nas características empreendedoras de alunos do curso de administração. Revista de Ciências da Administração, v. 1, n. 1, p. 99-111, 2015.

COLLIS, J; HUSSEY, R. Pesquisa em administração: um guia prático para alunos de graduação e pós-graduação. Bookman, 2005.
CORRAL-VERDUGO, V.; PINHEIRO, J. Q. Condições para o estudo do comportamento pró-ambiental. Estudos de Psicologia, v. 4, n. 1, p. 7-22, 1999.

DEGEN, R. J. Empreendedorismo: uma filosofia para o desenvolvimento sustentável e a redução da pobreza. Revista de Ciências da Administração, v. 10, n. 21, p. 11-30, 2008.

DE LEEUW, A.; VALOIS, P.; AJZEN, I.; SCHMIDT, P. Using the theory of planned behavior to identify key beliefs underlying pro-environmental behavior in high-school students: Implications for educational interventions. Journal of Environmental Psychology, v. 42 , p. 128-138, 2015.

DENTCHEV, N.; BAUMGARTNER, R.; DIELEMAN, H.; JÓHANNSDÓTTIR, L.; JONKER, J.; NYBERG, T.; VAN HOOF, B. Embracing the variety of sustainable business models: social entrepreneurship, corporate intrapreneurship, creativity, innovation, and other approaches to sustainability challenges. Journal of Cleaner Production, v. 113, p. 1-4, 2016.

FAYOLLE, A.; GAILLY, B. The impact of entrepreneurship education on entrepreneurial attitudes and intention: Hysteresis and persistence. Journal of Small Business Management, v. 53, n. 1, p. 7593, 2015.

FERREIRA, A. S. M.; LOIOLA, E.; GONDIM, S. M. G. Individual and contextual predictors of entrepreneurial intention among undergraduates: a literature review. Cadernos EBAPE. BR, v. 15, n. 2, p. 292-308, 2017.

FERREIRA, C. A.; SOARES, J. F; CRUZ, F. R. B. Reconhecimento de padrões em Estatística: Uma abordagem Comparativa. In: V Congresso Brasileiro de Redes Neurais. 2001. p. 409-414.

FOXALL, G. R.; HACKETT, P. M. W. The factor structure and construct validity of the Kirton AdaptionInnovation Inventory. Personality and Individual Differences, v. 13, n. 9, p. 967-975, 1992. 
GOLDSMITH, R. E.; KERR, J. R. Entrepreneurship and adaption-innovation theory. Technovation, v. 11, n. 6, p. 373-382, 1991.

GOMES, G.; GORNI, P. M.; DREHER, M. T. Consumo sustentável e o comportamento de universitários: discurso e práxis! RECADM, v. 10, n. 2, p. 80-92, 2011.

GORGIEVSKI, M. J.; STEPHAN, U.; LAGUNA, M.; MORIANO, J. A. Predicting entrepreneurial career intentions: Values and the theory of planned behavior. Journal of Career Assessment, v. 26, n. 3, p. $457-475,2018$.

GONÇALVES-DIAS, S. L. F.; TEODÓSIO, A. D. S. S.; CARVALHO, S.; SILVA, H. M. R. Consciência ambiental: um estudo exploratório sobre suas implicações para o ensino de administração. RAE-eletrônica, v. 8, n. 1, p. 1-23, 2009.

GRISKEVICIUS, V.; CANTÚ, S. M.; VUGT, M. V. The evolutionary bases for sustainable behavior: Implications for marketing, policy, and social entrepreneurship. Journal of Public Policy \& Marketing, v. 31, n. 1, p. 115-128, 2012.

GÜNTHER, H.; PINHEIRO, J. Q.; GUZZO, R. S. L. Psicologia Ambiental: entendendo as relações do homem com seu ambiente. Campinas: Alínea, 2004.

HAIR, J. F.; BLACK, W. C.; BABIN, B. J.; ANDERSON, R. E.; TATHAM, R. L. Análise multivariada de dados. Bookman Editora, 2009.

HAN, H.; KIM, Y. An investigation of green hotel customers' decision formation: Developing an extended model of the theory of planned behavior. International Journal of Hospitality Management, v. 29, n. 4 , p. 659-668, 2010.

HAUSER, J.; TELLIS, G. J.; GRIFFIN, A. Research on innovation: A review and agenda for marketing science. Marketing science, v. 25, n. 6, p. 687-717, 2006.
HOCKERTS, K. Determinants of social entrepreneurial intentions. Entrepreneurship Theory and Practice, v. 41, n. 1, p. 105-130, 2017.

HÖRISCH, J.; KOLLAT, J.; BRIEGER, S. A. What influences environmental entrepreneurship? A multilevel analysis of the determinants of entrepreneurs' environmental orientation. Small Business Economics, v. 48, n. 1, p. 47-69, 2017.

KAUTONEN, T.; VAN GELDEREN, M.; FINK, M. Robustness of the theory of planned behavior in predicting entrepreneurial intentions and actions. Entrepreneurship Theory and Practice, v. 39, n. 3, p. $655-674,2015$.

KHUONG, M. N.; AN, N. H. The factors affecting entrepreneurial intention of the students of Vietnam national university-a mediation analysis of perception toward entrepreneurship. Journal of Economics, Business and Management, v. 4, n. 2, p. 104-111, 2016.

KIRTON, M. Adaptors and innovators: A description and measure. Journal of Applied Psychology, v. 61, n. 5, p. $622,1976$.

KOLLMANN, T.; STÖCKMANN, C.; MEVES, Y.; KENSBOCK, J. M. When members of entrepreneurial teams differ: linking diversity in individual-level entrepreneurial orientation to team performance. Small Business Economics, v. 48, n. 4, p. 843-859, 2017.

KRUEGER, N. F.; CARSRUD, A. L. Entrepreneurial intentions: applying the theory of planned behaviour. Entrepreneurship \& Regional Development, v. 5, n. 4, p. 315-330, 1993.

KRUEGER, N. F. Entrepreneurial intentions are dead: Long live entrepreneurial intentions. In: Revisiting the Entrepreneurial Mind. Springer, Cham, 2017. p. 13-34.

KUCKERTZ, A.; WAGNER, M. The influence of sustainability orientation on entrepreneurial intentionsInvestigating the role of business experience. Journal of Business Venturing, v. 25, n. 5, p. 524-539, 2010. 
LIÑÁN, F.; CHEN, Y. W. Development and Cross $\$ Cultural application of a specific instrument to measure entrepreneurial intentions. Entrepreneurship theory and practice, v. 33, n. 3, p. 593-617, 2009.

MARCATI, A.; GUIDO, G.; PELUSO, A. M. The role of SME entrepreneurs' innovativeness and personality in the adoption of innovations. Research Policy, v. 37, n. 9, p. 1579-1590, 2008.

MUELLER, S. L.; DATO-ON, M. C. Gender-role orientation as a determinant of entrepreneurial self-efficacy. Journal of Developmental Entrepreneurship, v. 13, n. 01, p. 3-20, 2008.

MAROCO, J. Análise estatística: com utilização do SPSS. 2007.

NABI, G.; WALMSLEY, A.; LIÑÁN, F., AKHTAR, I.; NEAME, C. Does entrepreneurship education in the first year of higher education develop entrepreneurial intentions? The role of learning and inspiration. Studies in Higher Education, v. 43, n. 3, p. 452-467, 2018.

PAIVA, L. E. B.; LIMA, T. C. B.; SOUSA, E. S.; PONTES, E. S. Sustainability, innovation, and entrepreneurship: a research with administration and secretariat students. Revista de Negócios, v. 24, n. 3 , p. 7-26, 2019.

PAIVA, L. E.; SOUSA, E. S.; LIMA, T. C.; SILVA, D. D. Planned behavior and religious beliefs as antecedents to entrepreneurial intention: a study with university students. RAM. Revista de Administração Mackenzie, v. 21, n. 2, 2020.

PASSARO, R.; QUINTO, I.; THOMAS, A. The impact of higher education on entrepreneurial intention and human capital. Journal of Intellectual Capital, v. 19, n. 1, p. 135-156, 2018.

PAÇO, A. M. F.; FERREIRA, J. M.; RAPOSO, M.; RODRIGUES, R. G.; DINIS, A. Behaviours and entrepreneurial intention: Empirical findings about secondary students. Journal of International Entrepreneurship, v. 9, n. 1, p. 20-38, 2011.
PATO, C. M. L.; TAMAYO, A. A escala de comportamento ecológico: desenvolvimento e validação de um instrumento de medida. Estudos de psicologia, v. 11, n. 3, p. 289-296, 2006.

RANDERSON, K.; BETTINELLI, C.; FAYOLLE, A.; ANDERSON, A. Family

entrepreneurship as a field of research: Exploring its contours and contents. Journal of Family Business Strategy, v. 6, n. 3, p. 143-154, 2015.

RAUCH, A.; WIKLUND, J.; LUMPKIN, G. T.; FRESE, $M$. Entrepreneurial orientation and business performance: An assessment of past research and suggestions for the future. Entrepreneurship Theory and Practice, v. 33, n. 3, p. 761-787, 2009.

SALLIS, J. F; OWEN, N.; FISHER, E. Ecological models of health behavior. Health Behavior: Theory, Research, and Practice, v. 5, p. 43-64, 2015.

SÁNCHEZ, J. C. University training for entrepreneurial competencies: Its impact on intention of venture creation. International Entrepreneurship and Management Journal, v. 7, n. 2, p. 239-254, 2011.

SANTOS, P. M. F.; PORTO, R. B. A gestão ambiental como fonte de vantagem competitiva sustentável: contribuições da visão baseada em recursos e da teoria institucional. Revista de Ciências da Administração, v. 15, n. 35, p. 152-167, 2013.

SCHLAEGEL, C.; KOENIG, M. Determinants of Entrepreneurial Intent: A Meta-Analytic Test and Integration of Competing Models. Entrepreneurship Theory and Practice, v. 38, n. 2, p. 291-332, 2014.

\section{SHAPERO, A.; SOKOL, L. The social dimensions of} entrepreneurship. 1982.

SIEGER, P.; MINOLA, T. The family's financial support as a "Poisoned giff": A family embeddedness perspective on entrepreneurial intentions. Journal of Small Business Management, v. 55, p. 179-204, 2017. 
SOOMRO, B. A; SHAH, N. Developing attitudes and intentions among potential entrepreneurs. Journal of Enterprise Information Management, v. 28, n. 2, p. 304-322, 2015.

SOUSA, A. M. R.; BIZARRIA, F. P. A.; LIMA, A. O.; BRASIL, M. V. O.; FONTENELE, R. E. S. Sentimentos Topofílicos e suas Relações com a Atitude e a Intenção Empreendedoras. Revista Eletrônica de Ciência Administrativa, v. 16, n. 3, p. 233-253, 2017.

SOUZA, R. S.; SILVEIRA, A.; NASCIMENTO, S. Ampliando a mensuração da intenção empreendedora. Revista de Administração FACES Journal, v. 17, n. 2, p. 74-93, 2018.

STEINMETZ, H.; KNAPPSTEIN, M.; AJZEN, I.; SCHMIDT, P.; KABST, R. How effective are behavior change interventions based on the theory of planned behavior? Zeitschrift für Psychologie, v. 224, n. 3, p. 216-233, 2016.

STRAUGHAN, R. D.; ROBERTS, J.A. Environmental segmentation alternatives: a look at green consumer behavior in the new millennium. Journal of Consumer Marketing, v. 16, n. 6, p. 558-575, 1999.

STUM, J. Kirton's adaption-innovation theory: Managing cognitive styles in times of diversity and change. Emerging Leadership Journeys, v. 2, n. 1, p. 66-78, 2009.

SUBOTIC, M.; MARIC, M.; MITROVIC, S.; MESKO, $\mathrm{M}$. Differences between adaptors and innovators in the context of entrepreneurial potential dimensions. Kybernetes, v. 47, n. 7, p. 1363-1377, 2018.

TACONELI, C. A.; ZOCCHI, S. S.; DIAS, C. T. S. Extensões do algoritmo de árvores de classificação para a análise de dados categorizados multivariados utilizando coeficientes de dissimilaridade e entropia. Revista Brasileira de Biometria, p. 93-114, 2008.

TEIXEIRA, A. A. C; DAVEY, T. Attitudes of Higher Education students to new venture creation: a preliminary approach to the Portuguese case. Industry and Higher Education, v. 24, n. 5, p. 323-341, 2010.
TENTAMA, F.; PAPUTUNGAN, T.H.Entrepreneurial Intention of Students Reviewed from Self-Efficacy and Family Support in Vocational High School. International Journal of Evaluation and Research in Education, v. 8, n. 3, p. 557-562, 2019.

THOMPSON, E. R. Individual entrepreneurial intent: Construct clarification and development of an internationally reliable metric. Entrepreneurship Theory and Practice, v. 33, n. 3, p. 669-694, 2009.

TILLEY, F.; YOUNG, W. Sustainability Entrepreneurs. Greener Management International, n. 55, 2009.

VICENZI, S. E.; BULGACOV, S. Fatores motivadores do empreendedorismo e as decisões estratégicas de pequenas empresas. Revista de Ciências da Administração, v. 15, n. 35, p. 208-221, 2013.

WANG, D.; WANG, L.; CHEN, L. Unlocking the influence of family business exposure on entrepreneurial intentions. International Entrepreneurship and Management Journal, p. 1-24, 2017.

WANG, C. K.; WONG, P. K. Entrepreneurial interest of university students in Singapore. Technovation, v. 24, n. 2, p. 163-172, 2004.

WURTHMANN, K. Business students' attitudes toward innovation and intentions to start their own businesses. International Entrepreneurship and Management Journal, v. 10, n. 4, p. 691-711, 2014.

ZAMPETAKIS, L. A.; MOUSTAKIS, V. Linking creativity with entrepreneurial intentions: A structural approach. The International Entrepreneurship and Management Journal, v. 2, n. 3, p. 413-428, 2006.

ZAMPETAKIS, L. A.; BAKATSAKI, M.; LITOS, C.; KAFETSIOS, K. G.; MOUSTAKIS, V. Gender-based differential item functioning in the application of the theory of planned behavior for the study of entrepreneurial intentions. Frontiers in Psychology, v. 8, p. 451, 2017. 